\title{
Demonstration of the Presence of the “Deleted” MIR122 Gene in HepG2 Cells
}

\author{
Ibrahim A. Y. Hamad ${ }^{1,2}$, Yue Fei ${ }^{1}$, Anastasia Z. Kalea ${ }^{1}$, Dan Yin ${ }^{1,3}$, Andrew J. P. Smith ${ }^{1}$, \\ Jutta Palmen ${ }^{1}$, Steve E. Humphries ${ }^{1}$, Philippa J. Talmud ${ }^{1}$, Ann P. Walker ${ }^{1}$. \\ 1 Centre for Cardiovascular Genetics, BHF Laboratories, Institute of Cardiovascular Science, UCL, London, \\ WC1E 6JF, United Kingdom, 2 Medical Biochemistry Department, Alazhar University, Damietta, 34511, \\ Egypt, 3 Key Laboratory of Molecular Biophysics of the Ministry of Education, College of Life Science and \\ Technology, Center for Human Genome Research, Huazhong University of Science and Technology \\ (HUST), Wuhan, 430074, PR China \\ *ann.walker@ucl.ac.uk
}

\section{G openaccess}

Citation: Hamad IAY, Fei Y, Kalea AZ, Yin D, Smith AJP, Palmen J, et al. (2015) Demonstration of the Presence of the "Deleted" MIR122 Gene in HepG2 Cells. PLoS ONE 10(3): e0122471. doi:10.1371/ journal.pone.0122471

Academic Editor: Sebastien Pfeffer, French National Center for Scientific Research - Institut de biologie moléculaire et cellulaire, FRANCE

Received: August 14, 2014

Accepted: February 13, 2015

Published: March 26, 2015

Copyright: @ 2015 Hamad et al. This is an open access article distributed under the terms of the Creative Commons Attribution License, which permits unrestricted use, distribution, and reproduction in any medium, provided the original author and source are credited.

Data Availability Statement: All relevant data are within the paper and its Supporting Information files.

Funding: This study was supported by: a channel scheme studentship from the Egyptian Cultural and Educational Bureau (IAYH, APW), http://www.mohecasm.edu.eg/; A National Institute for Health Research University College London Hospitals Biomedical Research Centre \#HZHD (AZK), (http:// www.uclhospitals.brc.nihr.ac.uk); A BHF Intermediate Basic Science Research Fellowship FS/13/6/29977 (AJPS) and a BHF Programme Grant PG 008/08 (SEH, PJT), (www.bhf.org.uk). The funders had no

\section{Abstract}

MicroRNA 122 (miR-122) is highly expressed in the liver where it influences diverse biological processes and pathways, including hepatitis $C$ virus replication and metabolism of iron and cholesterol. It is processed from a long non-coding primary transcript ( 7.5 kb) and the gene has two evolutionarily-conserved regions containing the pri-mir-122 promoter and premir-122 hairpin region. Several groups reported that the widely-used hepatocytic cell line HepG2 had deficient expression of miR-122, previously ascribed to deletion of the pre-mir122 stem-loop region. We aimed to characterise this deletion by direct sequencing of 6078 bp containing the pri-mir-122 promoter and pre-mir-122 stem-loop region in HepG2 and Huh-7, a control hepatocytic cell line reported to express miR-122, supported by sequence analysis of cloned genomic DNA. In contrast to previous findings, the entire sequence was present in both cell lines. Ten SNPs were heterozygous in HepG2 indicating that DNA was present in two copies. Three validation isolates of HepG2 were sequenced, showing identical genotype to the original in two, whereas the third was different. Investigation of promoter chromatin status by FAIRE showed that Huh-7 cells had $6.2 \pm 0.19$ - and $2.7 \pm 0.01$ - fold more accessible chromatin at the proximal (HNF4a-binding) and distal DR1 transcription factor sites, compared to HepG2 cells ( $\mathrm{p}=0.03$ and 0.001 , respectively). This was substantiated by ENCODE genome annotations, which showed a DNAse I hypersensitive site in the pri-mir-122 promoter in Huh-7 that was absent in HepG2 cells. While the origin of the reported deletion is unclear, cell lines should be obtained from a reputable source and used at low passage number to avoid discrepant results. Deficiency of miR-122 expression in HepG2 cells may be related to a relative deficiency of accessible promoter chromatin in HepG2 versus Huh-7 cells. 
role in study design, data collection and analysis, decision to publish, or preparation of the manuscript.

Competing Interests: The authors have declared that no competing interests exist.

\section{Introduction}

MicroRNA 122 (miR-122, encoded by EntrezGene ID 406906; OMIM *609582) is predominantly expressed in the liver [1]. MicroRNAs generally act by binding to partially complementary sequences in the 3' untranslated region (UTR) of target mRNAs, resulting in either mRNA degradation or block of translation. However miR-122 may also act via a different mechanism, as it also binds to the 5' UTR of the RNA genome of the hepatitis C virus (HCV), promoting viral replication [2]. MiR-122 participates in regulation of post-transcriptional gene expression in diverse physiological and pathological processes. These include support of the HCV viral cycle in hepatocytes, cell growth, apoptosis, carcinogenesis and regulation of hepatic iron and cholesterol metabolism [1-7]. Thus, antagonism of miR-122 decreased total plasma cholesterol levels whereas miR-122-mimics were shown to target the HFE and HFE2 (HJV) genes, influencing hepcidin levels and thereby body iron absorption, in both animal models and man. A phase 2 clinical trial of miR-122 inhibition using the locked nucleic acid miR-122 inhibitor miravirsen for treatment of chronic HCV genotype 1 infection reported dose-dependent reductions in the level of HCV RNA. Serum total cholesterol levels were also decreased by 25\% over the 12 week period after miravirsen treatment [4-7]. Hence miR-122 may also be considered as a potential therapeutic target in non-alcoholic fatty liver disease, the metabolic syndrome and cardiovascular disease.

Reduced expression of miR-122 in hepatocellular carcinoma versus control liver tissue and implication of many of its target mRNAs in carcinogenesis suggested that miR-122 acts as a tumour suppressor in hepatocytes [8-10]. However, studies of the expression pattern of miR-122 in two models of human liver cancer reported that miR-122 is expressed in the Huh-7 cell line but deficient in HepG2 [3,9-13]. The potential basis of this difference in expression was investigated by cloning and sequencing the pre-mir-122 stem-loop genomic region, along with two sequences proposed at that time as putative promoter regions, from HepG2 and Huh-7 cells. The pre-mir-122 stem-loop region, but not the two upstream regions, was reported to be deleted only in HepG2 cells and this was suggested to be the cause of deficient miR-122 expression in these cells [9]. However, the results of the cloning experiment appeared inconclusive and the pri-mir-122 promoter was not investigated $[9,14]$. We therefore aimed to clarify the nature of any deletion of the MIR122 gene in the genomic DNA of the widely-used HepG2 hepatocytic model cell line.

\section{Materials and Methods}

HepG2 cells were obtained directly from European Collection of Cell Cultures (ECACC, Public Health England, Porton Down, England; catalogue no. 85011430, passage +101 , vial date $12 / 04 / 11$ ), where the identity of the cell line had been validated by short tandem repeat DNA profile and karyotype analyses. After 6 weeks storage in liquid nitrogen, the culture was established and after a single passage, the cell pellet was harvested for DNA preparation (HepG2-1). Validation isolate HepG2-2, from Dr Clare Selden, UCL (Royal Free Hospital Campus), UK was validated as HepG2 by analysis of both microsatellite genotypes and "DNA barcodes"; tested mycoplasma-free in three different assays; tested free of selected human pathogens and was shown to be sterile by direct inoculation analysis [15]. HepG2-3, from Professor Ann K. Daly, Newcastle University Medical School, UK, was originally obtained from ATCC. Details of the third laboratory isolate, HepG2-4, are available upon request. Control Huh-7 D12 cells (referred to as Huh-7; catalogue no. 01042712, passage 19) were obtained directly from the European Collection of Cell Cultures (Public Health England, Porton Down, England) [16,17]. HepG2 cells were cultured in Eagle's minimal essential medium with $10 \%$ foetal bovine serum (FBS) and 1\% non-essential amino acids (Thermo Scientific SH3023801) and 
Huh-7 cells in Dulbecco's Modified Eagle Medium with 10\% FBS and $2 \mathrm{mM}$ glutamine under $5 \% \mathrm{CO}_{2}$ at $37^{\circ} \mathrm{C}$. Cultures were passaged at $70-80 \%$ confluence and seeded at $2-3 \times 10^{6}$ cells per $75 \mathrm{~cm}^{2}$ flask.

Genomic DNA was extracted using the Nucleospin Blood QuickPure kit (Macherey-Nagel, Germany). Primers were designed using Primer3 (v. 0.4.0) [18,19] to amplify a 6078 bp region spanning the pri-mir-122 promoter and pre-mir-122 stem-loop region in 13 overlapping fragments for direct sequencing (Source Bioscience, Nottingham, UK; S1 Table) [9,14]. The sequence across a heterozygous, frame-shifting length polymorphism of a poly $(\mathrm{T})$ tract was investigated by amplification of the miR122_735F / miR122_735R fragment, initially using Taq PCR Master Mix Kit (QIAGEN, UK). Fragments were cloned into pGEM-T Easy Vector System (Promega, UK) and transformed into E. coli XL-10 Gold (Stratagene / Agilent Technologies, UK) for plasmid purification using QIAprep Spin Miniprep kit (QIAGEN, UK) and sequencing using vector SP6 and T7 RNA polymerase promoter primers. Three HepG2 and five Huh-7 clones were sequenced. The cloning was repeated using Phusion high fidelity DNA polymerase (New England Biolabs Ltd, UK) and cloning the PCR products into Zero Blunt TOPO (Life Technologies Ltd, UK), transformation into E. coli TOP10 for plasmid purification using the QIAprep Spin Miniprep kit and sequencing using vector T3 and T7 RNA polymerase promoter primers of seven HepG2 and eight Huh-7 clones. The sequences were aligned to the GRCh37/hg19 (Feb. 2009) human genome assembly using ClustalW [20]. The Encyclopedia of DNA Elements (ENCODE) annotations and the positions of common SNPs were identified using the UCSC Genome Browser and dbSNP [21-23]. The 6078 bp region spanning the primir-122 promoter and pre-mir-122 region was re-sequenced for validation in three additional HepG2 isolates from different sources. All primer sequences are listed in S1 Table.

Formaldehyde-Assisted Isolation of Regulatory Elements (FAIRE) was performed as previously described [24-26]. Sonication of genomic DNA from HepG2 and Huh-7 cells was performed using a UCD300 Bioruptor Next Generation sonication system (Diagenode s.a., Belgium) with a protocol of 20 cycles ( 1 min sonication, 1 min cooling; sonication level: high) at $4^{\circ} \mathrm{C}$, in $100-300 \mu \mathrm{l}$ volumes ( $1.5 \mathrm{ml}$ tubes), to obtain DNA fragments of $100-1000 \mathrm{bp}$, as determined by electrophoresis on $1 \%$ agarose, $0.5 \%$ Nusieve GTG agarose (Lonza) gels prepared in $8.9 \mathrm{mM}$ Tris-borate buffer, $2 \mathrm{mM}$ EDTA pH 8.0 (1x TBE). Quantitative real-time PCR (qRT-PCR) analysis was performed in a 384 well plate using 10 ng FAIRE or non-FAIRE control DNA as template, 1x ABI Power SYBR Green mastermix \#4367659 and $0.5 \mu \mathrm{M}$ forward and reverse primers in $10 \mu \mathrm{l}$ reactions. Primer pairs q122DR1-1F / q122DR1-1R and q122DR1-2F / q122DR1-2R (S1 Table) were designed for the proximal and distal DR-1 sites in the pri-mir-122 promoter, respectively [14]. Primers qHPRT1e3F / qHPRT1e3R were identified from ENCODE annotations to flank a well-defined, "gold standard" control site of inaccessible chromatin in HepG2 and Huh-7 cells under basal conditions, as recommended [27]. The qRT-PCR was performed using an ABI 7900HT Real-Time PCR System and the data were collected using RQ Manager v1.2 software. Three biological replicate cell culture experiments were analysed, each with four technical replicates. The comparative Ct method $\left(2^{-\Delta \Delta C t}\right)$ was used to calculate the relative level of accessible chromatin, as the proportion of that in HepG2 [28]. No template controls were run in each assay to verify absence of PCR contamination.

Data are presented as mean \pm SEM and significance was determined using the Students' paired, two tailed t-test.

\section{Results}

The pre-mir-122 stem-loop region, which was previously not detected in DNA from HepG2 cells and was reported likely to be mutated or deleted, was sequenced and shown to be present 


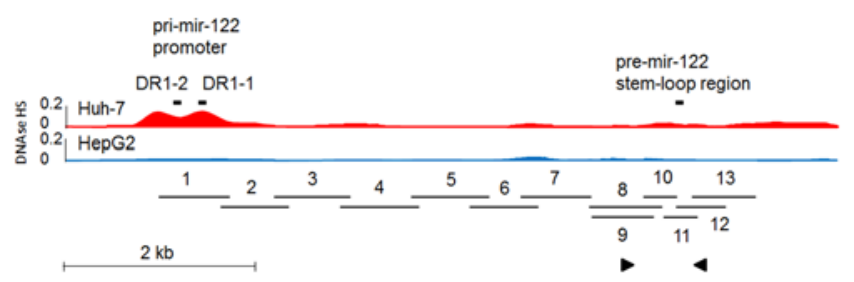

Fig 1. The "deleted" MIR122 gene is present in two copies in HepG2 cells. A schematic diagram illustrates the human MIR122 genomic locus. Li et al (2011) identified the pri-mir-122 promoter and the two DR-1 sites which were investigated by FAIRE in this study [14]. The position of the pre-mir-122 stem-loop region, previously reported to be deleted in HepG2 cells, is indicated. FAIRE showed that Huh-7 cells had $6.2 \pm 0.19$ - and $2.7 \pm 0.01$ - fold more accessible chromatin at the DR1-1 (proximal) and DR1-2 (distal) sites than HepG2 ( $p=0.03$ and 0.001$)$, respectively. The ENCODE DNase I hypersensitivity (DNAse HS, relative units; Duke University) annotations of MIR122 are shown as density signals for Huh-7 and HepG2 [22,23]. Consistent with the FAIRE results, these annotations showed a DNase I hypersensitivity site in the pri-mir122 promoter in Huh-7 that was not seen in HepG2. The region was sequenced in 13 reactions (overlapping horizontal bars) and shown to be present in both Huh-7 and HepG2 cell lines (HepG2 isolates 1, 2 and 3). Ten SNPs spanning the region were heterozygous indicating that DNA was present in two copies. The positions of the miR-122 gene (upstream) and miR-122 gene (downstream) primers (Table 1) previously used to report the HepG2 MIR122 "deletion" [9] are indicated by arrowheads; the downstream primer was located within an AluJb repeat sequence. The widely-reported deficiency of miR-122 expression in HepG2 cells is not caused by a MIR122 deletion, but may be related to a less accessible chromatin conformation in HepG2 than Huh-7 cells. Scale bar, $2 \mathrm{~kb}$.

doi:10.1371/journal.pone.0122471.g001

in the genomic DNA of HepG2 cells. The primer (miR-122 gene (downstream)) which Wu and colleagues previously used to report a deletion mapped within an Alu repeat sequence. This primer and its pair (miR-122 gene (upstream)) also contained one and three nucleotide differences, respectively, to the GRCh37 / hg19 (Feb. 2009) human genome assembly at their 5' ends (Fig. 1) [9]. Direct sequencing of 6078 bp of genomic DNA containing the pri-mir-122 promoter and the pre-mir-122 hairpin region indicated identical sequence to chr18:5611298356119060 of the GRCh37 / hg19 assembly, with the exception of 10 SNPs for which two different alleles were demonstrated in the original isolate HepG2-1, indicating that two copies of this genomic region were present in the genomic DNA of HepG2 cells (Fig. 1) [9,14,22]. Validation in three additional isolates of HepG2 cells showed identical genotype for the karyotyped- and "DNA barcoded"- reference isolate HepG2-2 and for HepG2-3, whereas HepG2-4 had a different genotype at all 10 SNPs (Table 1).

The results from cloned single allele sequencing from the original HepG2-1 isolate confirmed that two of the upstream SNPs, rs9966765 and rs1135519, were heterozygous. In addition, analysis of the cloned sequences for a poly $(\mathrm{T})$ length polymorphism located upstream of the pre-mir-122 stem-loop region (reported in dbSNP as rs143672020 and rs71173053) was consistent with the presence of two alleles for HepG2 (Fig. 2). The single allele cloned sequences obtained after amplification with Taq polymerase showed both an apparent slippage in the length of the poly $(\mathrm{T})$ tract and sequence differences between clones at positions not corresponding to the positions of common SNPs (Fig. 2A). The cloning was therefore repeated after amplification using a proofreading DNA polymerase. Such changes were still observed after amplification with Phusion High Fidelity DNA Polymerase, with 3/15 (20\%) of single allele clones obtained from HepG2 and Huh-7 DNA showing apparent poly(T) slippage and also four sequence variants observed that were not seen in other clones of the same haplotype (Fig. 2B). Overall, despite this sequence heterogeneity, the polymorphisms confirmed two different haplotypes in HepG2 DNA, consistent with the presence of two alleles of the pre-mir122 stem-loop region. 
Table 1. Sequence validation of HepG2 identity confirmed the presence and heterozygosity of the pri-mir-122 promoter and pre-mir-122 stem-loop region.

\begin{tabular}{|c|c|c|c|c|}
\hline \multirow[t]{2}{*}{ PCR } & \multirow[t]{2}{*}{ SNP } & \multicolumn{3}{|c|}{ Genotype of cell line isolate } \\
\hline & & HepG2-1, HepG2-2 \& HepG2-3 & HepG2-4 ${ }^{a}$ & Huh-7 \\
\hline 1 & rs7227488 & AG & AA & GG \\
\hline 1 & rs60575556 & $\mathrm{GG}$ & TT & GG \\
\hline 2 & rs4245271 & $A G$ & AA & GG \\
\hline 4 & rs4245272 & AT & $\mathrm{TT}$ & AA \\
\hline 4 & rs4940703 & $A G$ & AA & GG \\
\hline 4 & rs4940704 & $\mathrm{GG}$ & AA & GG \\
\hline 5 & rs9319929 & $A C$ & AA & $\mathrm{CC}$ \\
\hline $6 \& 7$ & rs9966765 & CG & $\mathrm{CC}$ & GG \\
\hline 7 & rs1135519 & CT & $\mathrm{CC}$ & $\mathrm{TT}$ \\
\hline $11,12 \& 13$ & rs17669 & CT & $\mathrm{CC}$ & $\mathrm{TT}$ \\
\hline $12 \& 13$ & rs145725411 & CT & $\mathrm{CC}$ & $\mathrm{TT}$ \\
\hline 13 & rs6566969 & $A G$ & AA & GG \\
\hline
\end{tabular}

The pre-mir-122 stem-loop region, previously reported to be deleted in HepG2, was shown to be present in all isolates sequenced (PCRs 10, 11 and 12). Ten SNPs were heterozygous in three isolates of HepG2, indicating that DNA was present in two copies.

${ }^{a}$ The genotype of the isolate designated HepG2-4 indicated that it was probably not HepG2.

doi:10.1371/journal.pone.0122471.t001

Chromatin accessibility in the original HepG2-1 isolate was analysed by FAIRE at the two DR-1 (potentially HNF4 $\alpha$-binding) transcription factor sites described in the pri-mir-122 promoter [14]. Consistent with the reported deficiency of miR-122 expression in HepG2 cells, FAIRE showed that Huh-7 cells had $6.2 \pm 0.19$ - and $2.7 \pm 0.01$ - fold more accessible chromatin at the proximal and distal DR-1 promoter sites, $(\mathrm{p}=0.03$ and 0.001 , respectively), than did HepG2 cells. Consistent with these findings, in silico analysis also indicated some regulatory differences between the two cell lines. A DNase I hypersensitive site was present at the pri-mir122 promoter in Huh-7 cells that was absent in the HepG2 cells, indicating a difference in potential promoter activity and suggesting an effect on miR-122 expression (Fig. 1) [14,22,23].

\section{Discussion}

MiR-122 is considered a potential therapeutic target as it regulates diverse processes and pathways, including $\mathrm{HCV}$ replication and hepatic iron and cholesterol metabolism. A clinical trial of miR-122 inhibition using miravirsen in the setting of $\mathrm{HCV}$ infection showed dose-dependent reduction of HCV RNA, along with a maximal reduction in serum total cholesterol concentration of $\sim 0.8-1.7 \mathrm{mM}$ and a sustained reduction 14 weeks after cessation of treatment of $\sim 0.4-$ $1.7 \mathrm{mM}$, depending on the dosage group [7]. The present study demonstrated that the region encompassing pri-mir-122 promoter and pre-mir-122 stem-loop was present in the HepG2 cell line, whereas deletion of the pre-mir-122 stem-loop region had been previously reported as the cause of low miR-122 expression in HepG2 cells [9,14]. This was supported by direct sequencing of PCR products and cloned DNA. Although "loss of heterozygosity" of tumour suppressor genes is a commonly observed step in tumourigenesis, there was no "loss of heterozygosity" of the MIR122 gene in HepG2 cells: two different alleles were detected at 10 SNPs spanning the 6078 bp genomic region spanning the pri-mir-122 promoter and the pre-mir-122 stem-loop region [29]. The sequence of the original isolate, obtained and used directly from ECACC, was identical to two of three additional isolates of HepG2 obtained from different laboratories, one of which had been validated as HepG2 by karyotyping and "DNA barcoding"[15]. The third 

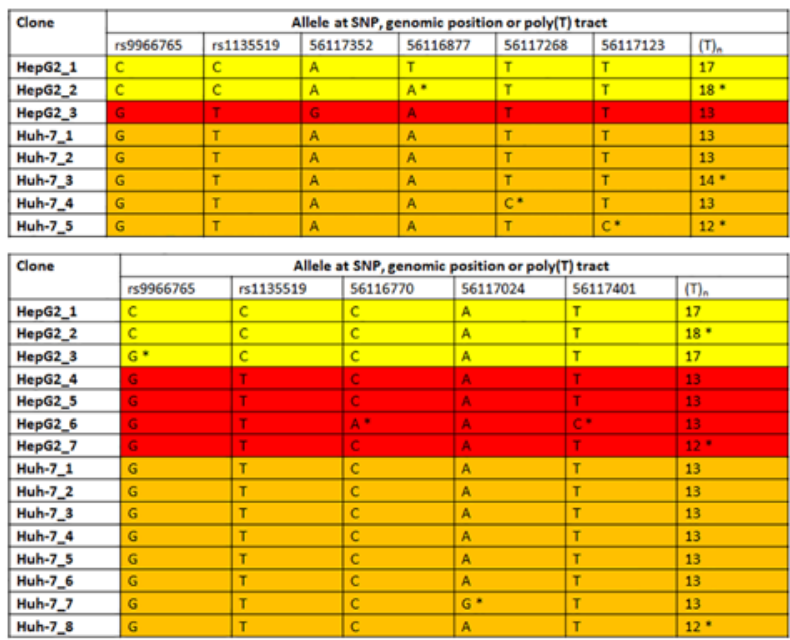

Fig 2. Cloned pre-mir-122 stem-loop region sequences from HepG2 DNA show two different haplotypes. (A) Cloned DNA sequences obtained after amplification with Taq polymerase. Two haplotypes (differently shaded) were observed for HepG2, consistent with the presence of two alleles across this region. However, among the eight HepG2 and Huh-7 clones, six sequence differences to the reference genome assembly were detected $\left({ }^{*}\right)$, so cloning was repeated using a proofreading DNA polymerase. (B) Cloned DNA sequences obtained after amplification with Phusion high fidelity DNA polymerase. Essentially the same two haplotypes of HepG2 were seen, but three novel single nucleotide substitution variants were detected and in a fourth clone, the rs 9966765 allele did not correspond to the background haplotype observed. The reported error rate of Phusion High-Fidelity DNA Polymerase (GC Buffer) is $9.5 \times 10^{-7}$ errors / base pair / PCR cycle (New England Biolabs). SNPs rs9966765 and rs 1135519 are located upstream of the pre-mir-122 stem-loop region; their respective alleles are shown. The genomic positions on chromosome 18 (GRCh37/ hg19 (Feb. 2009) human genome assembly) of non-SNP sequence variants and the alleles observed are shown; $(T)_{n}$ refers to the length (base pairs) of the polymorphic poly $(T)$ tract. *, position showing a sequence variant not corresponding to the predominant haplotypes observed.

doi:10.1371/journal.pone.0122471.g002

isolate also showed no evidence of deletion across the $6078 \mathrm{bp}$ region sequenced; however, it had a different genotype at all 10 SNPs, indicating that this cell line was probably not HepG2. This observation emphasises the importance of obtaining cell lines from a reputable source and using them at low passage number. Overall, the data confirmed the presence of the region spanning the pri-mir-122 promoter and pre-mir-122 stem-loop region in the genome of HepG2 cells. Thus, deletion is not the cause of the widely reported low basal miR-122 expression in HepG2 cells [3,9-13]. This deficient expression could therefore result from chromatin or histone features either of the MIR122 gene itself or of an enhancer or other remote regulatory sequence.

MiR-122 is predominantly expressed in the liver where it regulates several hepatic pathways and processes. The liver-enriched transcription factor HNF4 $\alpha$ is an important regulator of miR-122 expression; in Huh-7 cells, HNF4 $\alpha$ was previously shown to bind to the proximal conserved DR-1 site in the pri-mir-122 promoter [14]. The distal DR-1 site was not investigated. FAIRE was therefore used to investigate accessibility of chromatin at the two predicted DR1 sites in the pri-mir-122 promoter. By FAIRE, Huh-7 cells had $6.2 \pm 0.19$ - and $2.7 \pm 0.01$ - fold more accessible chromatin at the proximal and distal DR1 sites, respectively, compared to HepG2 cells ( $\mathrm{p}=0.03$ and 0.001 , respectively). Consistent with these findings, in silico analysis of ENCODE annotations showed a DNAse I hypersensitive site in the pri-mir-122 promoter of the Huh-7 cell line that was absent from HepG2 cells. This indicated that under the basal conditions studied, in Huh-7 cells, DNA at this site was relatively accessible for binding of transcription factors whereas in HepG2, chromatin was condensed and inaccessible. Taken together, these data indicate that chromatin at two predicted HNF4 $\alpha$ binding sites in the 
HepG2 promoter (one of which was previously demonstrated to bind HNF4 $\alpha$ ) is less accessible than that in Huh-7 cells, potentially leading to transcriptional repression. This mechanism could contribute to or explain the reported difference in miR-122 expression between the two cell lines.

Sequence heterogeneity of a poly(T) tract in the MIR122 gene was detected by sequencing cloned DNA, which also supported the presence of two different alleles in HepG2 cells, although some clone haplotypes showed an apparent "slippage" in the length of the poly(T) tract and / or additional "non-SNP" single nucleotide substitutions in individual clones. The origin of this micro-heterogeneity of DNA sequence is not known, but it could have been present in the original tumour from which the HepG2 cell line was derived [16], or may have occurred during cloning or propagation of the cell line, or as a result of PCR or cloning. Similar sequence heterogeneity was observed for cloned Huh-7 DNA. The observed error rate was $~ 14$-fold higher than that reported for Phusion High-Fidelity DNA Polymerase (GC Buffer) (New England Biolabs; Fig. 2) which, if this error rate is correct, suggests that these changes are unlikely to have arisen solely as a result of PCR errors. The spectral karyotype of HepG2 was previously reported to show widespread cytogenetic heterogeneity, with both aneuploidy and structural abnormalities of chromosomes (52-78, XY; $\operatorname{del}(1)(\mathrm{p} 22) ;+2 ;+\operatorname{der}(6) \mathrm{t}(6 ; 17)(\mathrm{p} 10 ; \mathrm{q} 22) ;+14$; $+\operatorname{der}(16) \mathrm{t}(6 ; 16)(\mathrm{q} 22 ; \mathrm{p} 13.3) ;+20 \mathrm{X} 2 ; \operatorname{der}(21) \mathrm{t}(1 ; 21)(\mathrm{p} 22 ; \mathrm{q} 10))$. However, these cytogenetic changes did not involve chromosome 18, which contains the MIR122 gene[30]. Other commonly used cell lines have been found to harbour gene deletions [31,32], underlining the value of confirming cell culture studies performed with easily manipulated cell lines in additional cell lines or in more physiologically-relevant systems such as primary cell cultures.

The original error leading to incorrect reporting of deletion of the pre-mir-122 stem-loop region [9] could have arisen due to failure of PCR amplification or cloning. The downstream primer used by these authors for PCR was within an Alu repeat, a family of highly repeated sequences that are estimated to be present in over a million copies in the human genome [33]. Hence, degeneracy of one PCR primer and primer-template mismatches could have contributed along with other factors, such as template quality, to failure of amplification of the pre-mir122 stem-loop region from HepG2 genomic DNA. Alternatively, the isolate of HepG2 cells used by these authors could possibly have undergone homozygous deletion of MIR122 if late passage number cells were used; or a failure of cloning could have led to the incorrect identification of an apparent deletion.

The HepG2 cell line has been widely used as model human hepatocytic cell line in many biological studies, with a PubMed search (HepG2; 02/03/15) showing 17,490 citations. HepG2 cells offer certain advantages over Huh-7 cells as a model system, such as the ability to polarise with tight junctions separating apical and basolateral poles, forming structures reminiscent of the bile canaliculi of intact liver. For example they have been proposed as a model in which to investigate the effects of hepatocyte polarity upon the HCV life cycle [34]. While HCV replication in the HepG2 cell line occurs at a level approximately 850 times lower than in Huh-7 cells, co-transfection of miR-122 and the HCV "receptor", CD81, into HepG2 enables these cells to support the entire viral life cycle [35]. Therefore, the demonstration that the entire MIR122 gene is present, and not deleted, is significant because it indicates the potential for induction of miR-122 expression in HepG2 cells by appropriate ligands, transcription factors and / or growth conditions.

In conclusion, contrary to the previously reported deletion, we found the entire locus spanning the pri-mir-122 promoter and pre-mir-122 stem-loop region is present in two copies in the widely used HepG2 cell line. Analysis of ENCODE DNAse I genome annotations and FAIRE investigation of HepG2 and Huh-7 cultures suggested that the reported lack of miR- 
122 expression in HepG2 cells may be related to repression of transcription by a relatively condensed chromatin structure within the pri-mir-122 promoter.

\section{Supporting Information}

S1 Table. Primers used for PCR, sequencing and qPCR. Bp, base pairs; Tm, the melting temperature used for PCR; n/a, not applicable. ${ }^{\text {aT }}$ These previously reported PCR primers were found to be problematic and the downstream primer mapped within an AluJb repeat sequence. (DOCX)

\section{Acknowledgments}

We thank Dr Clare Selden, UCL (Royal Free Hospital Campus), UK (HepG2-2) and Professor Ann K. Daly, Newcastle University Medical School, UK (HepG2-3), for kindly providing HepG2 validation isolates.

\section{Author Contributions}

Conceived and designed the experiments: IAYH APW. Performed the experiments: IAYH YF DY AJPS JP APW. Analyzed the data: IAYH YF AZK SEH PJT APW. Wrote the paper: IAYH AZK SEH AJPS JP APW.

\section{References}

1. Lagos-Quintana M, Rauhut R, Yalcin A, Meyer J, Lendeckel W, Tuschl T. Identification of tissue-specific micrornas from mouse. Curr Biol. 2002; 12: 735-739. PMID: 12007417

2. Henke JI, Goergen $D$, Zheng J, Song $Y$, Schuttler CG, Fehr $C$, et al. Microrna-122 stimulates translation of hepatitis c virus rna. EMBO J. 2008; 27: 3300-3310. doi: 10.1038/emboj.2008.244 PMID: 19020517

3. Chang J, Nicolas E, Marks D, Sander C, Lerro A, Buendia MA, et al. Mir-122, a mammalian liver-specific microrna, is processed from hcr mrna and may downregulate the high affinity cationic amino acid transporter cat-1. RNA Biol. 2004; 1: 106-113. PMID: 17179747

4. Castoldi M, Vujic Spasic M, Altamura S, Elmen J, Lindow M, Kiss J, et al. The liver-specific microrna mir-122 controls systemic iron homeostasis in mice. J Clin Invest. 2011; 121: 1386-1396. doi: 10.1172/ JCI44883 PMID: 21364282

5. Elmen J, Lindow M, Schutz S, Lawrence M, Petri A, Obad S, et al. Lna-mediated microrna silencing in non-human primates. Nature. 2008; 452: 896-899. doi: 10.1038/nature06783 PMID: 18368051

6. Esau C, Davis S, Murray SF, Yu XX, Pandey SK, Pear M, et al. Mir-122 regulation of lipid metabolism revealed by in vivo antisense targeting. Cell Metab. 2006; 3: 87-98. PMID: 16459310

7. Janssen HL, Reesink HW, Lawitz EJ, Zeuzem S, Rodriguez-Torres M, Patel K, et al. Treatment of hcv infection by targeting microrna. N Engl J Med. 2013; 368: 1685-1694. doi: 10.1056/NEJMoa1209026 PMID: 23534542

8. Bai S, Nasser MW, Wang B, Hsu SH, Datta J, Kutay H, et al. Microrna-122 inhibits tumorigenic properties of hepatocellular carcinoma cells and sensitizes these cells to sorafenib. J Biol Chem. 2009; 284: 32015-32027. doi: 10.1074/jbc.M109.016774 PMID: 19726678

9. Wu X, Wu S, Tong L, Luan T, Lin L, Lu S, et al. Mir-122 affects the viability and apoptosis of hepatocellular carcinoma cells. Scand J Gastroenterol. 2009; 44: 1332-1339. doi: 10.3109/00365520903215305 PMID: 19891584

10. Fornari F, Gramantieri L, Giovannini C, Veronese A, Ferracin M, Sabbioni S, et al. Mir-122/cyclin g1 interaction modulates p53 activity and affects doxorubicin sensitivity of human hepatocarcinoma cells. Cancer Res. 2009; 69: 5761-5767. doi: 10.1158/0008-5472.CAN-08-4797 PMID: 19584283

11. Jopling CL, Yi M, Lancaster AM, Lemon SM, Sarnow P. Modulation of hepatitis $c$ virus rna abundance by a liver-specific microrna. Science. 2005; 309: 1577-1581. PMID: 16141076

12. Murakami Y, Yasuda T, Saigo K, Urashima T, Toyoda H, Okanoue T, et al. Comprehensive analysis of microrna expression patterns in hepatocellular carcinoma and non-tumorous tissues. Oncogene. 2006; 25: 2537-2545. PMID: 16331254 
13. Basu S, Bhattacharyya SN. Insulin-like growth factor-1 prevents mir-122 production in neighbouring cells to curtail its intercellular transfer to ensure proliferation of human hepatoma cells. Nucleic Acids Res. 2014; 42: 7170-7185. doi: 10.1093/nar/gku346 PMID: 24813441

14. Li ZY, Xi Y, Zhu WN, Zeng C, Zhang ZQ, Guo ZC, et al. Positive regulation of hepatic mir-122 expression by hnf4alpha. J Hepatol. 2011; 55: 602-611. doi: 10.1016/j.jhep.2010.12.023 PMID: 21241755

15. Selden C, Spearman CW, Kahn D, Miller M, Figaji A, Erro E, et al. Evaluation of encapsulated liver cell spheroids in a fluidised-bed bioartificial liver for treatment of ischaemic acute liver failure in pigs in a translational setting. PLoS One. 2013; 8: e82312. doi: 10.1371/journal.pone.0082312 PMID: 24367515

16. Aden DP, Fogel A, Plotkin S, Damjanov I, Knowles BB. Controlled synthesis of hbsag in a differentiated human liver carcinoma-derived cell line. Nature. 1979; 282: 615-616. PMID: 233137

17. Cheng D, Yang A, Thomas $\mathrm{H}$, Monjardino J. Characterization of stable hepatitis delta expressing hepatoma cell lines: Effect of hdag on cell growth. Prog Clin Biol Res. 1993; 382: 149-153. PMID: 8502673

18. Koressaar $\mathrm{T}$, Remm M. Enhancements and modifications of primer design program primer3. Bioinformatics. 2007; 23: 1289-1291. PMID: 17379693

19. Untergasser A, Cutcutache I, Koressaar T, Ye J, Faircloth BC, Remm M, et al. Primer3-new capabilities and interfaces. Nucleic Acids Res. 2012; 40: e115. PMID: 22730293

20. Larkin MA, Blackshields G, Brown NP, Chenna R, McGettigan PA, McWilliam H, et al. Clustal w and clustal x version 2.0. Bioinformatics. 2007; 23: 2947-2948. PMID: 17846036

21. Sherry ST, Ward MH, Kholodov M, Baker J, Phan L, Smigielski EM, et al. Dbsnp: The ncbi database of genetic variation. Nucleic Acids Res. 2001; 29: 308-311. PMID: 11125122

22. Kent WJ, Sugnet CW, Furey TS, Roskin KM, Pringle TH, Zahler AM, et al. The human genome browser at ucsc. Genome Res. 2002; 12: 996-1006. PMID: 12045153

23. Bernstein BE, Birney E, Dunham I, Green ED, Gunter C, Snyder M. An integrated encyclopedia of DNA elements in the human genome. Nature. 2012; 489: 57-74. doi: 10.1038/nature11247 PMID: 22955616

24. Giresi PG, Kim J, McDaniell RM, lyer VR, Lieb JD. Faire (formaldehyde-assisted isolation of regulatory elements) isolates active regulatory elements from human chromatin. Genome Res. 2007; 17: 877-885. PMID: 17179217

25. Simon JM, Giresi PG, Davis IJ, Lieb JD. Using formaldehyde-assisted isolation of regulatory elements (faire) to isolate active regulatory DNA. Nat Protoc. 2012; 7: 256-267. doi: 10.1038/nprot.2011.444 PMID: 22262007

26. Smith AJ, Howard P, Shah S, Eriksson P, Stender S, Giambartolomei C, et al. Use of allele-specific faire to determine functional regulatory polymorphism using large-scale genotyping arrays. PLoS Genet. 2012; 8: e1002908. doi: 10.1371/journal.pgen.1002908 PMID: 22916038

27. Harbers M, Kahl G. Tag-based next generation sequencing: Wiley. 2012

28. Livak KJ, Schmittgen TD. Analysis of relative gene expression data using real-time quantitative pcr and the 2(-delta delta $\mathrm{c}(\mathrm{t}))$ method. Methods. 2001; 25: 402-408. PMID: 11846609

29. Knudson AG. Hereditary cancer: Two hits revisited. J Cancer Res Clin Oncol. 1996; 122: 135-140. PMID: 8601560

30. Wong N, Lai P, Pang E, Leung TW, Lau JW, Johnson PJ. A comprehensive karyotypic study on human hepatocellular carcinoma by spectral karyotyping. Hepatology. 2000; 32: 1060-1068. PMID: 11050057

31. Vecchi C, Montosi G, Pietrangelo A. Huh-7: A human "hemochromatotic" cell line. Hepatology. 2010; 51: 654-659. doi: 10.1002/hep.23410 PMID: 20017200

32. Lewis NE, Liu X, Li Y, Nagarajan H, Yerganian G, O'Brien E, et al. Genomic landscapes of chinese hamster ovary cell lines as revealed by the cricetulus griseus draft genome. Nat Biotechnol. 2013; 31 759-765. doi: 10.1038/nbt.2624 PMID: 23873082

33. Houck CM, Rinehart FP, Schmid CW. A ubiquitous family of repeated DNA sequences in the human genome. J Mol Biol. 1979; 132: 289-306. PMID: 533893

34. Decaens $\mathrm{C}$, Durand $\mathrm{M}$, Grosse $\mathrm{B}$, Cassio $\mathrm{D}$. Which in vitro models could be best used to study hepatocyte polarity? Biol Cell. 2008; 100: 387-398. doi: 10.1042/BC20070127 PMID: 18549352

35. Narbus CM, Israelow B, Sourisseau M, Michta ML, Hopcraft SE, Zeiner GM, et al. Hepg2 cells expressing microrna mir-122 support the entire hepatitis c virus life cycle. J Virol. 2011; 85: 12087-12092. doi: 10.1128/JVI.05843-11 PMID: 21917968 